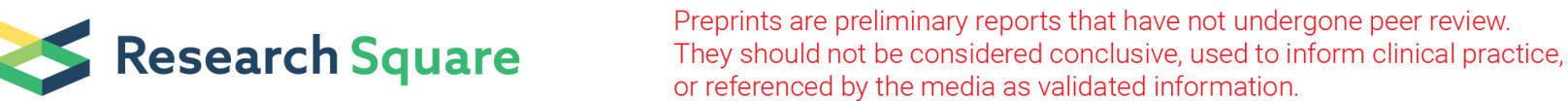

\section{Three kinds of treatment with Homoharringtonine, Hydroxychloroquine or shRNA and their combination against coronavirus PEDV in intro}

\section{Cui-Cui Li}

China Agricultural University https://orcid.org/0000-0003-0508-8956

Xiao-Jia Wang ( $\sim$ wangxj@cau.edu.cn )

\section{Research}

Keywords: coronavirus, PEDV, short hairpin RNA, heptad repeat, homoharringtonine, hydroxychloroquine, combination

Posted Date: May 21st, 2020

DOI: https://doi.org/10.21203/rs.2.23990/v2

License: (c) (i) This work is licensed under a Creative Commons Attribution 4.0 International License. Read Full License

Version of Record: A version of this preprint was published at Virology Journal on June 3rd, 2020. See the published version at https://doi.org/10.1186/s12985-020-01342-w. 


\section{Abstract}

Background: Porcine epidemic diarrhea virus (PEDV) of the family Coronaviridae has caused substantial economic losses in the swine husbandry industry. There's currently no specific drug available for treatment of coronaviruses or PEDV.

Method: In the current study, we use coronavirus PEDV as a model to study antiviral agents. Briefly, a fusion inhibitor tHR2, recombinant lentivirus-delivered shRNAs targeted to conserved $\mathrm{M}$ and $\mathrm{N}$ sequences, homoharringtonine $(\mathrm{HHT})$, and hydroxychloroquine (HCQ) were surveyed for their antiviral effects.

Results: Treatment with $\mathrm{HCQ}$ at $50 \mathrm{mM}$ and $\mathrm{HHT}$ at $150 \mathrm{nM}$ reduced virus titer in $\mathrm{TCID}_{50}$ by 30 and 3.5 fold respectively, and the combination reduced virus titer in $\mathrm{TCID}_{50}$ by 200 fold.

Conclusion: Our report demonstrates that the combination of HHT and HCQ exhibited higher antiviral activity than either HHT or HCQ exhibited. The information may contribute to the development of antiviral strategies effective in controlling PEDV infection.

\section{Introduction}

Porcine epidemic diarrhea virus (PEDV) belongs to the subfamily Coronavirinae of the family Coronaviridae, which causes epidemic diarrhea in swine, and was first reported in 1971 in the United Kingdom and was soon identified in many European and Asian countries [1]. The incidences of diarrhea occurred in suckling piglets are $80 \%$ to $100 \%$ on piggeries with an outbreak in China and Southeast Asia [2-3]. At present, there's no treatment for PEDVavailable.

The current strategies for controlling viral infectivity focus on identification of agents capable of intervening in the essential steps for viral infection, especially viral entry and replication. The PEDV is an enveloped, positive-sense single-strand RNA virus. The viral genome is approximately $28 \mathrm{~kb}$ and includes more than seven open reading frames (ORF) that encode 4 structural proteins, including spike glycoprotein $(S)$, nucleocapsid protein $(N)$, membrane protein $(M)$, and envelope protein $(E), 15$ nonstructural proteins (NSP), and accessory protein ORF3 [1]. N protein is a phosphorylated nucleocapsid protein, and the epitope of $\mathrm{N}$ protein can induce the body to produce an effective immune response [4]. $\mathrm{M}$ protein plays an essential role in viral assembly [5]. In RNA interference (RNAi), short interfering RNA molecules or short hairpin RNA (shRNA) targeting specific mRNA leads to degradation of mRNA and inhibition of gene expression [6-7]. RNAi targeting viral genes is an effective way to prevent cells from virus infection, and has been shown to mediate genetic suppression of many human and animal pathogenic coronaviruses $[8,9,10]$. Heptad repeat $(H R)$ regions are highly conserved motifs located in spike glycoprotein of enveloped viruses. The viruses such as coronavirus utilize a conserved membrane fusion mechanism, in which highly conserved heptad repeat (HR) regions interact to form six-helix bundle (6-HB) structures, contributing to the fusion of virus and cell membranes. The HIV-1 fusion inhibitor T20, for example, and several modified peptides (e.g., T1249, T2635, T-20 $\mathrm{s}_{138 \mathrm{~A}}$ ), which are derived from the HR region, have been clinically approved to suppress HIV-1 and T-20-resistant variants [11-14]. 
Coronavirus S glycoprotein shares a mechanism similar to other Class I virus glycoproteins for membrane fusion [15-17]. Our previous work has shown that the modified HR peptide efficiently blocks coronavirus infection $[17,18]$.

Viruses are able to utilize cellular machineries for viral replication, and a growing understanding of cellular proteins and associated pathways in viral replication has supported the design of new strategies for developing novel antiviral agents. Small molecule chemicals that are able to target cellular machinery act as efficient antiviral agents. The natural compound homoharringtonine (HHT), for instance, is known to inhibit the first cycle of the elongation phase of eukaryotic translation. HHT antagonizes the phosphorylation level of endogenous and exogenous elF4E ( $p$-elF4E), which may regulate the selective translation of specific mRNA $[19,20]$. In a previous study, we observed that HHT completely inhibited infection by PEDV at a concentration of $500 \mathrm{nM}$ in cell cultures; treatment with $\mathrm{HHT}$ at doses of 0.05 $\mathrm{mg} / \mathrm{kg}$ when after the infection significantly reduced viral load (in the intestine and blood) and relieved severe symptoms in PEDV-infected piglets [20]. One crucial cellular mechanism of autophagy, relies on lysosomes for the clearance and recycling of abnormal proteins or organelles; the deregulation of autophagy is associated with the development of various diseases, including viral infection by PEDV coronavirus [21]. Hydroxychloroquine (HCQ), an autophagy inhibitor chloroquine hydroxy-derivative, acts as an anti-inflammatory agent by attenuating tissue injury through downregulation of inflammatory activation [22]. It also triggers the host defense machinery by inducing ROS- and MAVS-mediated innate immune activation [23]. HCQ shows antiviral activity against a broad spectrum of viruses, including Zika virus and Dengue virus $[23,24]$.

In the present study, we constructed a truncated HR2 peptide and recombinant lentivirus-delivered shRNAs targeted to conserved $\mathrm{M}$ and $\mathrm{N}$ sequences, as well as small molecule chemicals $\mathrm{HHT}$ and $\mathrm{HCQ}$, to evaluate their antiviral activity. We identified that the combinations of HHT and HHR2 or HCQ more significantly improved the antiviral effects compared to the action of individual components. This paper provides evidence of the potential in vitro antiviral effects against coronaviruses.

\section{Materials And Methods}

\section{Cells, virus and antibodies}

Vero cells were cultured in Dulbecco's modified Eagle's medium (DMEM) supplemented with $2 \mathrm{mM} \mathrm{L-}$ glutamine, nonessential amino acids, sodium pyruvate, $5 \%$ or $10 \%$ heat-inactivated fetal bovine serum (FBS), $100 \mathrm{U} / \mathrm{ml}$ penicillin and $100 \mu \mathrm{g} / \mathrm{ml}$ streptomycin (all reagents are purchased from Gibco Invitrogen). Cells were cultured at $37^{\circ} \mathrm{C}$ in a humidified incubator with $5 \% \mathrm{CO}_{2}$. PEDV strain $\mathrm{CV} 777$ stock with $1 \times 10^{7} / \mathrm{ml}$ plaque forming units (PFU) is used in this paper. Anti PEDV-N (1:1000) mouse monoclonal antibody was obtained from Alpha Diagnostic International. Antibodies to actin (1:1000) and goat anti-mouse secondary antibodies conjugated to horseradish peroxidase (HRP, 1:10000) were obtained from Beyotime Biotechnology. 


\section{Design of shRNA plasmids}

The shRNA expression vector pGPU6/GFP/Neo was selected to be the parental plasmid. The shRNA sequences were as follows (5'-3'; only the sense strand is shown). shRNA-N: GCAAAGACTGAACCCACTA; shRNA-M: CTGGAATTTCACATGGAAT. The shRNA-MN plasmid carrying two shRNAs was prepared based on the two intermediate plasmids shRNA-N and shRNA-M. Briefly, shRNA-MN DNA sense template was Bbs I- (shRNA-N) - hU6 promoter- (shRNA-M) - BamH I. Empty vector pGPU6/GFP/Neo and shRNA-MN sequence were digested with $B b s$ I and $B a m H I$ respectively. Then the shRNA-MN sense was ligased to the vector pGPU6/GFP/Neo by T4 DNA ligase. All of these nucleotides were chemically synthesized by Invitrogen.

\section{Transfection of shRNA plasmids and virusinfection}

For transfection experiments, $2 \times 10^{5}$ Vero cells were cultured in six-well plates to $50-70 \%$ confluence. Cells were transfected with plasmid using TransIntro EL according to the manufacturer's protocols. Briefly, for each well, $4 \mu \mathrm{g}$ shRNA plasmid were incubated with $100 \mu$ opti-MEM for $5 \mathrm{~min}$, and $10 \mu \mathrm{l}$ TransIntro ELwere incubated with $100 \mu \mathrm{l}$ opti-MEM for $5 \mathrm{~min}$. Subsequently, mixture of $10 \mu \mathrm{l}$ Transintro EL and $100 \mu \mathrm{l}$ opti-MEM was added to the mixture of plasmid and opti-MEM. After incubation for 10-20 min at room temperature (RT), the mixture was added to the well. After incubation at $37^{\circ} \mathrm{C}$ for $5 \mathrm{~h}$, the medium containing the transfection mix was replaced with growth medium (DMEM-2\%FBS). At 24 h posttransfection (h.p.t), cells were infected with PEDV at multiplicity of infection (MOI) of 0.1. Cultures were then incubated at $37^{\circ} \mathrm{C}, 5 \% \mathrm{CO}_{2}$ in a humidified incubator for $1.5 \mathrm{~h}$, at which point the medium was replaced with growth medium. After $48 \mathrm{~h}$ of incubation, cells were havested to detect virus titers and the levels of mRNA.

\section{HHT and HCQ treatment and viral infection}

For HCQ, Vero cells were treated with HCQ $(25 \mathrm{mM}$ and $50 \mathrm{mM})$ at the time of PEDV infection (0.1MOI) for $1 \mathrm{~h}$ at $37^{\circ} \mathrm{C}$, washed three times with PBS, and overlaid with DMEM-2\% FBS containing HCQ with indicated concentrations. At 24 h.p.i., cells were harvested for WB assay and mRNA was extracted for RTPCR. For HHT, Vero cells were infected with PEDV $(0.1 \mathrm{MOI})$ for $1 \mathrm{~h}$ at $37^{\circ} \mathrm{C}$, washed three times with PBS, and overlaid with DMEM-2\% FBS containing HHT with indicated concentrations. At 48 h.p.i., cells were harvested for WB assay and $\mathrm{TCID}_{50}$ assay. For combination of HCQ and HHT experiment, Vero cells were infected with PEDV (0.1MOI) in the absence or presence of HCQ $(25 \mathrm{mM}$ and $50 \mathrm{mM})$ for $1 \mathrm{~h}$ at $37^{\circ} \mathrm{C}$, washed three times with PBS, and overlaid with DMEM-2\% FBS in the absence or presence of HHT or HCQ with indicated concentrations. At 48 h.p.i., cells were harvested for $\operatorname{TCID}_{50}$ assay.

\section{MTT assay}

The MTT assay was conducted according to the manufacturer's (Beyotime Biotechnology) protocol. Briefly, cells in 96 -well plates were treated with shRNA at $37^{\circ} \mathrm{C}$ and $5 \% \mathrm{CO}_{2}$ for $24 \mathrm{~h}$. Then, $10 \mu \mathrm{l}$ of MTT solution was added to each well and the samples were incubated for $4 \mathrm{~h}$. The medium was then removed 
and $100 \mu \mathrm{l}$ of Formazan solution was added to each well. Optical density (OD) values were measured at a test wave length of $570 \mathrm{~nm}$ using a Mullikan Ascent Microplate Photometer (Thermo Fisher Scientific, Waltham, MA, USA).

\section{Preparation of cell lysates and western blotting}

The cells in 6-well plate were collected by centrifugation, washed three times with PBS and dissolved in $200 \mu$ lysis buffer (pH7.5 20 mM Tris-HCl, 150 mM NaCl, 1\% Triton X-100, 1 mM EDTA, 2.5 mM Sodium pyrophosphate, $1 \mathrm{mM} \beta$-Glycerrophosphate, $1 \mathrm{mM} \mathrm{NaVO4,} 1 \mu \mathrm{g} / \mathrm{ml}$ Leupeptin) containing protease inhibitor cocktail (Roche) for $30 \mathrm{~min}$ on ice. The cell suspension was then fractionated by centrifugation at $6000 \times \mathrm{g}$ for $10 \mathrm{~min}$ at $4^{\circ} \mathrm{C}$. Solubilized proteins were harvested, quantified by BCA assay, electrophoresed in denaturing polyacrylamide gels (with $40 \mu \mathrm{g} / \mathrm{well}$ ), electro blotted onto a polyvinylidene fluoride (PVDF) membrane, and the antibodies detected the viral protein PVDF. Protein bands were detected with secondary antibody conjugated to horseradish peroxidase (HRP) for 45 min at room temperature, and actin was used as a loading control.

\section{Quantitative real-time PCR (qRT-PCR)}

Cells were harvested, and total RNA was extracted with Trizol (Invitrogen). The RNA was reversetranscribed into complementary DNA (cDNA). A two-step RT-PCR (SYBR Green I technology, Applied Roche) was performed using SYBR green super mix (Toyobo) according to the manufacturer's protocol to measure transcription levels for several genes of interest. The primers used were as follows: PEDV-N: 5'CTG GGT TGC TAA AGA AGG CG -3' (forward), 5'- CTG GGG AGC TGT TGA GAG AA -3' (reverse). IL-1b: 5'GAC CTG GAC CTC TGC CCT CTG-3' (forward), 5'- AGG TAT TTT GTC ATT ACT TTC-3' (reverse). IL-6: 5'AAC TCC TTC TCC ACA AGC -3' (forward), 5'- TGG ACT GCA GGA ACT CCT -3' (reverse). GAPDH: 5'-GAT CAT CAG CAA TGC CTC CT -3' (forward), 5'- TGA GTC CTT CCA CGA TAC CA -3' (reverse).

Relative fold changes were automatically calculated by the Step One Plus real-time PCR system software (Applied Bio systems), following the $2^{-\Delta \Delta C T}$ method. GAPDH as a control.

\section{Determination of $50 \%$ tissue culture infectious dose $\left(\mathrm{TCID}_{50}\right)$}

Vero cells in 6-well plates were cultured overnight with $80 \%$ confluency. Serial dilutions of the PEDV were added to the cells and let them infect of 90 min, and then the culture supernate was replaced with $2 \%$ FBS of DMEM and incubated for $48 \mathrm{~h}$. Virus titration was quantified. Ten-fold serial dilutions were prepared for each sample and $100 \mathrm{ml} /$ well of each dilution were added to the cells in 96-well plates in quadruplicates. Wells with cytopathic effect were scored as positive for virus growth and TCID $_{50}$ was determined by the method of Reed and Muench.

\section{Prediction and construction of HR1 and tHR2}

The software packages LearnCoil-VMF (http://nightingale.lcs.mit.edu) and ExPASy-Coils (http://www.ch.embnet.org/software/COILS) were used to study the coiled coils. HR1 and HR2 domains 
of spike glycoprotein (gS) from the PEDV (GeneBank Accession No. ALS35469.1), derived from amino acids 978 to 1118 and 1263 to 1314 (52Aa), respectively, were predicted. In addition, an optimised Lupas algorithm was used with window widths of 14,21 and 28 and the MTIDK matrix. The predicted probability of forming coiled coils was 0.9 . Optimal focused HR1 and HR2 domains were predicted as follows: NSAIGNITSA FESVKEA ISQTSKGL NTVAH ALTKVQEVVN SQGSALNQLT VQLQHNFQAI, and LTGE IADLEQR SESLRNT TEELRSL INNINNT LVDLEWL (39Aa), respectively. The scramble peptide is LTGE IADVEQR SESVRNT TEEVRSL INNVNNT LVDVEWL, which is also derived from the PEDV HR2 domain, as negative control (NC). All of these peptides were chemically synthesized by China Peptides (http://www.chinapeptides.com/english/index.aspx).

\section{Gel-filtration analysis}

The highly purified HR1 and tHR2 were loaded onto the Superdex G75 column in a solution buffer of 20 $\mathrm{mM}$ Tris- $\mathrm{HCl}, \mathrm{pH}$ 8.0. The peak MW was estimated by comparing the substrate with protein standards running on the same column. The analytical column was calibrated with a series of individual runs of standard molecular mass proteins as markers, including bovine serum albumin (68 kDa), egg white albumin (43 kDa), ribose nucleotides (13.7 kDa), and antimicrobial peptides (5 kDa).

\section{Circular dichroism (CD) spectra analysis}

Peptides were dissolved in PBS ( $\mathrm{pH} 7.4)$ at a concentration of $30 \mu \mathrm{M}$. The wavelength-dependence of molar ellipticity [q] was monitored at $25^{\circ} \mathrm{C}$ using an average of eight scans in a spectropolarimeter (Model J-710, JASCO) with 0.2-cm path length cuvettes from 195-245 nm, equipped with a thermoelectric temperature controller. The buffers were filtered in a vacuum pump system with $0.2-\mu \mathrm{m}$ pore membrane filters. Routine calibration of the machine was performed with d-10 camphor sulphonic acid (60 mg 100 $\mathrm{ml}^{-1}$ ) according to the equation $[\mathrm{Q}]=100 \mathrm{Q} \mathrm{cnl}{ }^{-1}$, where $\mathrm{Q}$ is the ellipticity (mdeg), $\mathrm{c}$ is the peptide concentration $(\mathrm{mM}), \mathrm{n}$ is the number of residues, and $\mathrm{I}$ is the path length $(\mathrm{cm})$. Data analyses and acquisition were performed using the spectra manager software provided by the equipment manufacturer. On average, three scans were taken with a scanning rate of $200 \mathrm{~nm} \mathrm{~min}{ }^{-1}$. The results were expressed as the mean residue ellipticity [Q] $\left(10^{-3}\right.$ degree $\left.\mathrm{cm}^{2} \mathrm{dmol}^{-1}\right)$. A CD spectra analysis was performed to study the secondary structural changes in single peptides and any combination of two peptides (peptides mixtures) at an equimolar concentration in PBS. In these studies, single peptides were prepared at a concentration of $30 \mu \mathrm{M}$, and peptide mixtures at an equimolar concentration in a constant volume (for example, for each peptide mixture, the final concentration of each peptide was $15 \mu \mathrm{M}$ ).

\section{Immunofluorescence assay}

HCQ treatment of PEDV-infected Vero cells (Fig. 3C) growing on glass coverslips in 24-well plates were fixed with $4 \%$ paraformaldehyde for 15 min, permeabilized with $0.1 \%$ Triton X-100 for 15 min,and blocked with phosphate-buffered saline (PBS) containing $10 \%$ horse serum plus $1 \%$ bovine serum albumin (BSA) (purchase from Solarbio life sciences) for $2 \mathrm{~h}$. The cells were washed three times with PBS and incubated with diluted primary antibodies overnight at $4^{\circ} \mathrm{C}$. Cells were washed with PBS three times at room 
temperature (RT), then incubated with fluorochrome-conjugated secondary antibodies in the dark for $1 \mathrm{~h}$ at RT. The cells were then rinsed by PBS, mounted on glass slide and examined; images were captured using an microscope Olympus FluoViewÔ FV1000. The primary antibody to PEDV-N (1:1000) and the goat anti-mouse lgG $(\mathrm{H}+\mathrm{L})$ secondary antibody conjugated to Alexa Flour 488 (Invitrogen).

\section{Statistics}

All results were expressed as means and standard deviations (SD). Statistical analyses were performed using Prism 5.01 (GraphPad Software). Significance was determined by one-way analysis of variance (ANOVA) with Dennett's multiple-comparison test.

\section{Results}

\section{Dual shRNA-expressing plasmid shRNA-MN was constructed}

To suppress the replication of PEDV, Vero cells were transfected with shRNA-expressing plasmid targeting the M (shRNA-M) or N (shRNA-N) of PEDV, or shRNA targeting the $M$ and $N$ genes (shRNA-MN). Here, for the dual shRNA expression vector, two shRNAs respectively targeting $M$ and $N$ were incorporated into one vector, pGPU6/GFP/Neo-shRNA-MN, which carries two expression cassettes each containing a U6 promoter. The two corresponding single shRNA-expressing plasmids, pGPU6-shRNA-M and pGPU6shRNA-N, were constructed and used in parallel (Fig. 1A). Transfection efficiency of shRNA-expressing plasmid shRNA-M was observed by vector GFP fluorescent label (Fig. 1B), and no measurable decrease in cell viability was detected (Fig. 1C).

\section{ShRNA-MN exhibited prophylactic antiviral activity against PEDV infection in Vero cells}

Two shRNAs respectively targeting the PEDV $\mathrm{M}$ and $\mathrm{N}$ genes, and one dual shRNA-expressing plasmid shRNA-MN, were transfected into Vero cells for $24 \mathrm{~h}$ before infection to assess the effect of shRNAexpressing plasmids on the replication of PEDV. PEDV-CV777 induced cytopathic effect at $48 \mathrm{~h}$ post infection (h.p.i.), while the effect was significantly reduced in shRNA-MN treated cells (Fig. 1D). After 48 h.p.i with PEDV, we observed a significant reduction in viral protein levels (PEDV-N) (Fig. 1E) under treatment with shRNA-N and shRNA-MN, but not with single shRNA-M plasmid. The mRNA levels were significantly inhibited when treated with shRNA-N or shRNA-MN (Fig. 1F). Furthermore, shRNA-MN or shRNA-N reduced 12-fold viral production (Fig. 1G), indicating that the antiviral effect of dual shRNAexpressing plasmids was the same as single shRNA-N.

\section{HR1 and tHR2 formed a heterotrimer structure}

The primary structure of each a-helix consists of a heptad repeat composed of seven residues commonly denoted by $(a-b-c-d-e-f-g)_{n}$. HR sequences were divided into two characteristic interactive sites $(a, d$,

$e)$ and solvent-accessible sites $(b, c, f, g)$. The predicted HR2 domain is derived from amino acids 1263 to 1314 (52Aa). Here we truncated the HR2 domain to produce the peptide tHR2 (1278-1316, 39Aa); the 
relevant helical structures are shown in Fig. 2A.HR1 and tHR2 were produced. The scramble peptide is LTGE IADVEQR SESVRNT TEEVRSL INNVNNT LVDVEWL, which is also derived from the PEDV HR2 domain, as negative control (NC). Determining any direct interaction between HR1 and tHR2, we mixed HR1 and tHR2 peptides, each at $15 \mathrm{mM}$, and incubated the mixture for $2 \mathrm{~h}$. Using a Superdex $\mathrm{G} 75$ column (GE Healthcare), we detected $34 \mathrm{kDa}$ polymers in the chromatographic elutes. The molecular weight (MW) of the HR1 domain from PEDV was approximately $6.5 \mathrm{kDa}$, and HR2 was approximately $4.3 \mathrm{kDa}$ (Fig. 2B). The $34 \mathrm{kDa}$ polymer may consist of three HR1 and three tHR2 peptides (Fig. 2B). HR1 and scrambled (NC) peptide were not able to bind each other (not shown here). This result indicates that HR1 and tHR2 interacted with each other. Our CD spectroscopic analysis of conformational changes in peptide mixtures revealed a distinguishable value in the HR1 and tHR2 mixture from individual peptides (Fig. 2D), indicating that interaction between HR1 and tHR2 peptides resulted in a different conformation from the homogenous polymers of either peptides. Detecting the effects of tHR2 on cell viability, we treated Vero cells with various concentrations of tHR2 peptide. The results showed that tHR2 peptide did not induce any changes of cell viability (Fig. 2 G).

\section{Peptide tHR2 exhibits antiviral activity against PEDV infection in Vero cells}

To test whether the peptides affected virus infection cycle, Vero cells were infected with PEDV at MOI of 0.1 for $48 \mathrm{~h}$ in the presence of tHR2 or NC peptide. We found that tHR2 clearly inhibited the expression of viral protein at $80 \mu \mathrm{M}$ (Fig. 2E), and it also reduced virus titer in $\mathrm{TCID}_{50}$ by approximately 4 fold (Fig. 2F). Inhibition by NC peptide was not observed (Figs. 2E, 2F). Taken together, these results show that exogenous tHR2 showed effective fusion inhibition activity, via competition for the endogenous HR1 region to block six-helix bundle structure formation.

\section{HCQ regulates autophagy and inflammatory response against PEDV infection}

LC3B is associated with autophagosome development and is used to monitor autophagic activity. Cleavage of PARP is a characteristic of apoptosis. Considering that autophagy is triggered in Vero cells during PEDV infection to promote its replication [21], we explored the effect of autophagy inhibitor HCQ on PEDV infection. As shown in Fig. 3A, HCQ reduced viral infection by blocking autophagy activation induced by PEDV in a dose-dependent manner; it seems that HCQ improved apoptosis via depressing autophagy. RT-qPCR analysis of cells treated with HCQ $(50 \mu \mathrm{M})$ showed decreased expression of proinflammatory cytokines IL-1 b and IL-6 (Fig. 3B). Vero cells were infected with PEDV of $0.1 \mathrm{MOI}$ in the presence of HCQ at 25 and $50 \mathrm{mM}$ for $24 \mathrm{~h}$. An indirect immunofluorescence assay was further carried out to verify the antiviral activities of HCQ, and result indicated that HCQ at $25 \mathrm{mM}$ reduced about $90 \%$ virions and almost completely inhibited PEDV replication at $50 \mathrm{mM}$ compared to DMSO (Fig. 3C). These results indicate that HCQ reduces PEDV-induced autophagy and inflammatory response. Effects of HCQ on the viability of Vero cells were determined by a MTT assay. Changes in cell viability was not detectable (Fig. 3D). 
Whether a combination of these agents will be optimal to control various viruses remains to be determined. Here, a gradual reduction in PEDV-N protein levels was observed when cells were treated with increasing doses of HHT (Fig. 4A). That is, HHT at $150 \mathrm{nM}$ reduced PEDV virus titer in $\mathrm{TCID}_{50}$ by 3.5 fold, and at $300 \mathrm{nM}$ reduced virus titer by approximately 40 fold (Fig. 4B). To further optimize the effects of the antivirals indicated above, the combination of tHR2 at $40 \mathrm{mM}$ and $80 \mathrm{mM}$ with HHT at $150 \mathrm{nM}$ was designed. Treatment of Combination of HHT and tHR2 when during and after the infection resulted in an additive effect on suppression (10-fold of $\mathrm{TCID}_{50}$ ) of viral production/infection at 48 h.p.i (Fig. $4 \mathrm{C}$ ). For combination of HCQ and HHT experiment, Vero cells were treated with HCQ (50 mM) during infection for $1 \mathrm{~h}$ at $37^{\circ} \mathrm{C}$, washed three times with PBS, and overlaid with DMEM-2\% FBS in the absence or presence of $\mathrm{HHT}$ or HCQ with indicated concentrations. At 48 h.p.i., cells were harvested for $\mathrm{TCID}_{50}$ assay. Notably, $\mathrm{HCQ}$ at $50 \mathrm{mM}$ reduced PEDV virus titer in $\mathrm{TCID}_{50}$ by 30 fold, and the combination with HHT at $150 \mathrm{nM}$ has addictive effects on infection and reduces TCID $_{50}$ by 200 fold. We studied if HHT or combined tHR2 with HCQ was able to modulate Vero cell viability. The results did not reveal any effects all HHT and combinations with tHR2 and HCQ on cell viability (Fig. 4E to 4G). These findings demonstrate that effectively inhibits PEDV infection, and the combination treatment at low concentrations produced significantly improved antiviral effect as compared to the separate components.

\section{Discussion}

In the current study, we found that the combination of HHT (150 nM) and HCQ at low concentrations (50 $\mathrm{mM}$ ) reduced $\mathrm{TCID}_{50}$ by 200 fold (Fig. 4). In our previous study, we found that HHT exerted broadspectrum antiviral action and completely inhibited infection by PEDV at a concentration of $500 \mathrm{nM}$ in Vero cells [20]. Treatment with HHT at doses of $0.05 \mathrm{mg} / \mathrm{kg}$ when after the infection significantly reduced viral load (in the intestine and blood) and relieved severe symptoms in PEDV-infected piglets, but treatment with $0.2 \mathrm{mg} / \mathrm{kg}$ of HHT led to severe side effects, fatal to about $50 \%$ of the piglets [20]. The inhibitory effect of HCQ is associated with host defense machinery and immune regulation; HCQ at high concentration ( $>20 \mathrm{mM}$ ) also exhibits cell toxicity [21-24]. The study of preparations therefore provides new ideas, but the damage of host cells resulting from increasing doses is a problem that cannot be ignored.

Recent research has shown that combination therapy may be more effective than monotherapy. IFN- $\beta$ in combination with ribavirin act highly synergistically on production of infectious virus titres and may be highly effective not only as prophylactic agent but also for the treatment of already infected SARS patients [25]. A combination of RNAi and a virus receptor trap exerts additive antiviral activity in coxsackievirus B3-induced myocarditis in mice [26]. Likewise, anti-TNF antibody administered with Sunitinib significantly reduces vascular leakage and synergizes to provide superior protection from lethal DENV infection compared with either agent alone in vivo[27]. Combination therapies targeting viruses at different stages of their infection cycle are a common strategy to improve the efficiency of the antiviral treatment, and the combination of multi-target preparations is expected to be less cytotoxic [28]. 
The RNAi-based strategy has different mechanisms of action than antibodies, small molecules, and other protein drugs. It is worth mentioning that the RNAi drug Onpattro (patisiran) was the first FDA-approved for adult patients with peripheral neuropathy (polyneuropathy) caused by hereditary transthyretin amyloidosis (hATTR) [29]. Methods to express multiple shRNAs from a single vector have been explored, including expression from multiple cassettes using the same promoter [30], or from a single vector containing multiple promoters [31]. The inhibition of viruses by means of RNAi is an approach to antivirals that has gained importance in recent years [10,32-35], but the use of multiple shRNAs in the elimination of viral infection has been less successful. In this paper, we established the dual shRNA expression vector, targeting $\mathrm{M}$ and $\mathrm{N}$ genes, which carries two U6 promoters. We have established a concept for developing transfection vectors that may have wide applications in gene antiviral strategies, including the delivery of siRNA to combat viral infection. This approach has the potential to be utilized for the prevention and control of coronavirus PEDV [36]. However, our study demonstrates that the antiviral effect of dual shRNA-expressing plasmids was the same as single shRNA-N. This result may be related with low transcription efficiency or low antiviral activity of shRNA-M in dual shRNA-expressing plasmid. Therefore, we think double shRNA needs to be improved for high transcription efficiency and antiviral activity. In the future, the antiviral effects of shRNA will be evaluated in vivo. It is proposed that porcine U6 promoter-based shRNA vector might have more transcriptional activity than human U6 promoter in piglet.

The membrane fusion process has been considered as a significant antiviral target and has received wide attention; the recombinant HR2 region of fusion glycoprotein is the ideal fusion inhibitor for class I enveloped viruses [11]. Based on a LearnCoil-VMF prediction result, amino acid L1252 to L1286 (35Aa) from the HR2 domain of S glycoprotein was selected to design a fusion inhibitor that efficiently blocks MERS-CoV entry [16-17]. The predicted HR2 domain (65Aa) from S glycoprotein of PEDV, however, is longer than that in MERS-CoV and other coronaviruses [16-18, 37], and synthetic HR2 peptide at the concentration of $40 \mu \mathrm{M}$ statistically inhibits PEDV infection [38]. Reducing the length of the active peptide may be an important consideration to reduce the cost of peptide agents. Here we truncated the tHR2 domain to produce the peptide tHR2 (39Aa), with the aim of identifying a minimal domain capable of disrupting the formation of the six-helix bundle required for viral cell entry. Our results suggest that tHR2, but not scrambled peptide, has the ability to inhibit PEDV infection (Figs. 3E, 3F). Furthermore, the antiviral effect of tHR2 is not as strong as expected when compared to recombinant HR2 peptides (as shown in [17]), perhaps due to the short length or disrupted core structure. It is noteworthy that by 2013 the FDA had already approved the listing of more than 60 peptide drugs [39]. Peptides will be widely used in medicine and biotechnology in the near further.

In this study, we explored how to maximize the antiviral effect of HHT while reducing host cell toxicity. We found that tHR2 peptide designed based on PEDV S genes effectively inhibits the membrane fusion of PEDV when added during and after the infection, via competition for the endogenous HR1 region to block six-helix bundle structure formation and does not affect the cell viability. The combination treatment with HHT when added after the infection and tHR2 when added during and after the infection results in additive protective effects by inhibiting membrane fusion and viral replication. We also found that the 
combination of HHT when added after the infection and pre-transfected shRNA-MN did not exhibit improved effects (not shown here), perhaps because they have the same target in the viral replication process. Short hairpin RNA (shRNA) targeting specific mRNA leads to degradation of mRNA and inhibition of gene expression and HHT may regulate the selective translation of specific mRNA to inhibit viral gene expreesion. We propose that they may have same target during process of gene express. The combination treatment with HHT when added after the infection and HCQ when added during and after the infection results in additive protective effects. The combination of a low dose (150 nM) of HHT and/or HCQ as host targeting antivirals (HTA) with tHR2 as direct-acting antiviral (DAA) has the potential to improve safety and increase the antiviral effect. These results provide a reference for the development of clinical drugs against coronavirus infection in the future.

\section{Conclusion}

This paper provides information to contribute to the development of effective antiviral strategies against PEDV coronavirus. Our proof-of-concept study identified the combination of HHT with HCQ at low concentration as having an significant high antiviral effectiveness. The combination exerts additive effects and is significantly more effective than either of the treatments separately in inhibiting PEDV infection in vitro.

\section{Declaration}

\section{Ethics approval and consent to participate}

Not applicable.

\section{Consent to publication}

Not applicable.

\section{Availability of data and materials}

All data generated or analyzed during this study are included in this published article.

\section{Competing interests}

The authors declare that they have no conflict of interest. None of the authors of this article has a financial or personal relationship with other people or organizations that could inappropriately influence or bias the content of the paper.

\section{Funding}

This work was supported by the National Natural Science Foundation of China (31772739), and by The National Key R \& D Program of China (2017YFD0502205, 2017YFD0502306). 


\section{Author's contribution}

Cui-Cui Li designed and performed the experiments, and analyzed the data.

Xiao-Jia Wang designed the experiments and wrote the manuscript.

Corresponding author: Xiao-Jia Wang, wangxj@cau.edu.cn.

\section{Acknowledgements}

Not applicable.

\section{Abbreviation}

h.p.i.: Hour post infection

HCQ: Hydroxychloroquine

HHT: Homoharringtonine

HR: Conserved heptad repeat regions

PEDV: Porcine epidemic diarrhea virus

PFU: Plaque forming units

RNAi: RNA interference

RT: Room temperature

SD: standard deviations

ShRNA: Short hairpin RNA

\section{References}

1. Song D, Park B. Porcine epidemic diarrhoea virus: a comprehensive review of molecular epidemiology, diagnosis, and vaccines. Vírus Genes 44:167-175(2012).

2. Pan $\mathrm{Y}$, Tian $\mathrm{X}, \mathrm{Li}$ W, Zhou $\mathrm{Q}$, Wang $\mathrm{D}, \mathrm{Bi} \mathrm{Y}$, et al. Isolation and characterization of a variant porcine epidemic diarrhea virus in China. Viral J 9: 195(2012).

3. Sun RQ, Cai RJ, Chen YQ, Liang PS, Chen DK, Song CX. Outbreak of porcine epidemic diarrhea in suckling piglets, China. Emerg Infect Dis 18: 161-163(2012).

4. Shi D, Shi H, Sun D, Chen J, Zhang X, Wang X, et al. Nucleocapsid interacts with NP, and protects it from proteolytic cleavage, enhancing cell survival, and is involved in PEDV growth. Sci Rep 7: 39700(2017). 
5. Fan J, Li Y. Cloning and sequence analysis of the $\mathrm{M}$ gene of porcine epidemic diarrhea virus $\mathrm{Ljb} / 03$. Virus Genes 30(1): 69-73(2005).

6. Ma Y, Chan CY, He ML. RNA interference and antiviral therapy. World J Gastroenterol 13: 51695179(2007).

7. Travella S, Keller B. Down-regulation of gene expression by RNA-induced gene silencing. Methods Mol Biol 478: 185-199 (2009).

8. Li CC, Dong HJ, Wang P, Meng W, Han SJ, Ning S, et al. Cellular protein GLTSCR2: a valuable target for the development of broad-spectrum antivirals. Antivir Res 142(6): 1-11(2017).

9. Zhou J, Huang F, Hua X, Cui L, Zhang W, Shen Y, et al. Inhibition of porcine transmissible gastroenteritis virus (TGEV) replication in mini-pigs by shRNA. Virus Res 149(1): 51-55(2010).

10. Wu CJ, Huang HW, Liu CY, Hong CF, Chan YL. Inhibition of SARS-CoV replication by siRNA. Antiviral Res 65(1): 45-48(2005).

11. Eckert DM, Kim PS. Mechanisms of viral membrane fusion and its inhibition. Ann Rev Biochem 70: 777-810(2001).

12. Eggink D, Langedijk JP, Bonvin AM, Deng Y, Lu M, Berkhout $B$, et al. Detailed mechanistic insights into HIV-1 sensitivity to three generations fusion inhibitors. J Biol Chem 284: 26941-26950(2009).

13. Nishikawa H, Nakamura S, Kodama E, Ito S, Kajiwara K, Izumi K, et al. Electrostatically constrained alpha-helical peptide inhibits replication of HIV-1 resistant to enfuvirtide. Int J Biochem Cell Biol 4: 891-899(2009).

14. Pan C, Liu S, Jiang S. HIV-1 gp41 fusion intermediate: a target for HIV therapeutics. J Formos Med Assoc 109: 94-105(2010).

15. Bosch BJ, van der Zee R, de Haan CA, Rottier PJ. The coronavirus spike protein is a class I virus fusion protein: structural and functional characterization of the fusion core complex. $J$ Virol 77 : 8801-8811(2003).

16. Gao J, Lu G, Qi J, Wu Y, Deng Y, Geng H, et al. Structure of the fusion core and inhibition of fusion by a heptad repeat peptide derived from the $S$ protein of Middle East respiratory syndrome coronavirus. J Virol 87(24): 13134-13140(2013).

17. Wang XJ, Li CG, Chi XJ, Wang M. Characterisation and evaluation of antiviral recombinant peptides based on the heptad repeat regions of NDV and IBV fusion glycoproteins. Virology 416: 65-74(2011).

18. Li CG, Tang W, Chi XJ, Dong ZM, Wang XX, Wang Cholesterol tag at N-terminal of the relatively broad spectrum of fusion inhibitory-peptide targets earlier stage of fusion glycoprotein activation and increases peptide's antiviral potency in vivo. J Virol 87:9223-9232(2013).

19. Zhou, H, Xu, RZ, Gu, Y, Shi, PF, Qian, S. Targeting of phospho-elF4E by homoharringtonine eradicates a distinct subset of human acute myeloid leukemia. Leuk Lymphoma 15: 1-13(2018).

20. Dong HJ, Wang ZH, Meng W, Li CC, Hu YX, Zhou L, et al. The natural compound homoharringtonine presents broad antiviral activity in vitro and in vivo. Viruses 10 (11): 601 (2018). 
21. Guo X, Zhang M, Zhang X, Tan X, Guo H, Zeng W, et al., Porcine epidemic diarrhea virus induces autophagy to benefit its replication. Viruses 9(3):53 (2017).

22. Tang TT, Lv LL, Pan MM, Wen Y, Wang B, Li ZL, et al. Hydroxychloroquine attenuates renal ischemia/reperfusion injury by inhibiting cathepsin mediated NLRP3 inflammasome activation. Cell Death Dis 9(3):351(2018).

23. Wang LF, Lin YS, Huang NC, Yu CY, Tsai WL, Chen JJ, et al. Hydroxychloroquine-inhibited dengue virus is associated with host defense machinery. J Interferon Cytokine Res 35(3):143-56(2015).

24. Kumar A, Liang B, Aarthy M, Singh SK, Garg N, Mysorekar I, et al. Hydroxychloroquine inhibits Zika virus NS2B-NS3 protease. ACS Omega 3(12):18132-18141 (2018).

25. Morgenstern B, Michaelis M, Baer PC, Doerr HW, Cinatl J. Ribavirin and interferon- $\beta$ synergistically inhibit SARS-associated coronavirus replication in animal and human cell lines. Biochem Biophys Res Commun 326: 905-8(2005). Stein EA, Pinkert S, Becher PM, Geisler A, Zeichhardt H, Klopfleisch R, et al. Combination of RNA interference and virus receptor trap exerts additive antiviral activity in coxsackievirus B3-induced myocarditis in mice. J Infect Dis211(4): 613-22 (2015).

26. Branche E, Tang WW,Viramontes KM, Young MP, Sheets N, Joo Y, et al. Synergism between the tyrosine kinase inhibitor sunitinib and anti-TNF antibody protects against lethal dengue infection. Antivir Res158(10):1-10 (2018).

27. Boutimah F, Eekels JJ, Liu YP, Berkhout B. Antiviral strategies combining antiretroviral drugs with RNAi-mediated attack on HIV-1 and cellular co-factors. Antivir Res 98: 121-129 (2013).

28. Sheridan M H. Patisiran: First Global Approval. Drug 78 (15):1625-1631(2018)

29. Ter Brake O, Konstantinova P, Ceylan M, Berkhout B. Silencing of HIV-1 with RNA interference: a multiple shRNA approach. Mol Ther 14: 883-892 (2006).

30. Hinton TM, Doran TJ. Inhibition of chicken anaemia virus replication using multiple short-hairpin RNAs. Antivir Res 80: 143-149 (2008).

31. Jain B, Jain A. Taming influenza virus: role of antisense technology. Curr Mol Med 15: 433-445 (2015).

32. Xu YF, Shen HY, Zhao MQ, Chen LJ, Li YG, Liao M, et al. Adenovirus-vectored shRNAs targeted to the highly conserved regions of VP1 and 2B in tandem inhibits replication of foot-and-mouth disease virus both in vitro and in vivo. J Virol Methods 181(1): 51-58 (2012).

33. Achazi K, Patel P, Paliwal R, Radonić A, Niedrig M, Donoso-Mantke O. RNA interference inhibits replication of tick-borne encephalitis virus in vitro. Antivir Res.93(1): 94-100 (2012).

34. Shen H, Zhang C, Guo P, Liu Z, Zhang J. Effective inhibition of porcine epidemic diarrhea virus by RNA interference in vitro. Virus Genes 51(2): 252-259(2015).

35. Wang Z, Ren L, Zhao X, et al. Inhibition of severe acute respiratory syndrome virus replication by small interfering RNAs in mammalian cells.J Virol78:7523 (2004).

36. Li CG, Tang W, Chi XJ, Dong ZM, Wang XX, Wang XJ. Cholesterol tag at N-terminal of the relatively broad spectrum of fusion inhibitory-peptide targets earlier stage of fusion glycoprotein activation 
and increases peptide's antiviral potency in vivo. J Virol 87: 9223-9232(2013).

37. Zhao $P$, Wang $B$, Ji CM, Cong $X$, Wang $M$, Huang YW. Identification of a peptide derived from the heptad repeat 2 region of the porcine epidemic diarrhea virus (PEDV) spike glycoprotein that is capable of suppressing PEDV entry and inducing neutralizing antibodies. Antivir Res 150: 1-8(2017).

38. Kaspar AA, Reichert JM. Future directions for peptide therapeutics development. Drug Discov Today 18(17-18): 807(2013).

\section{Figures}
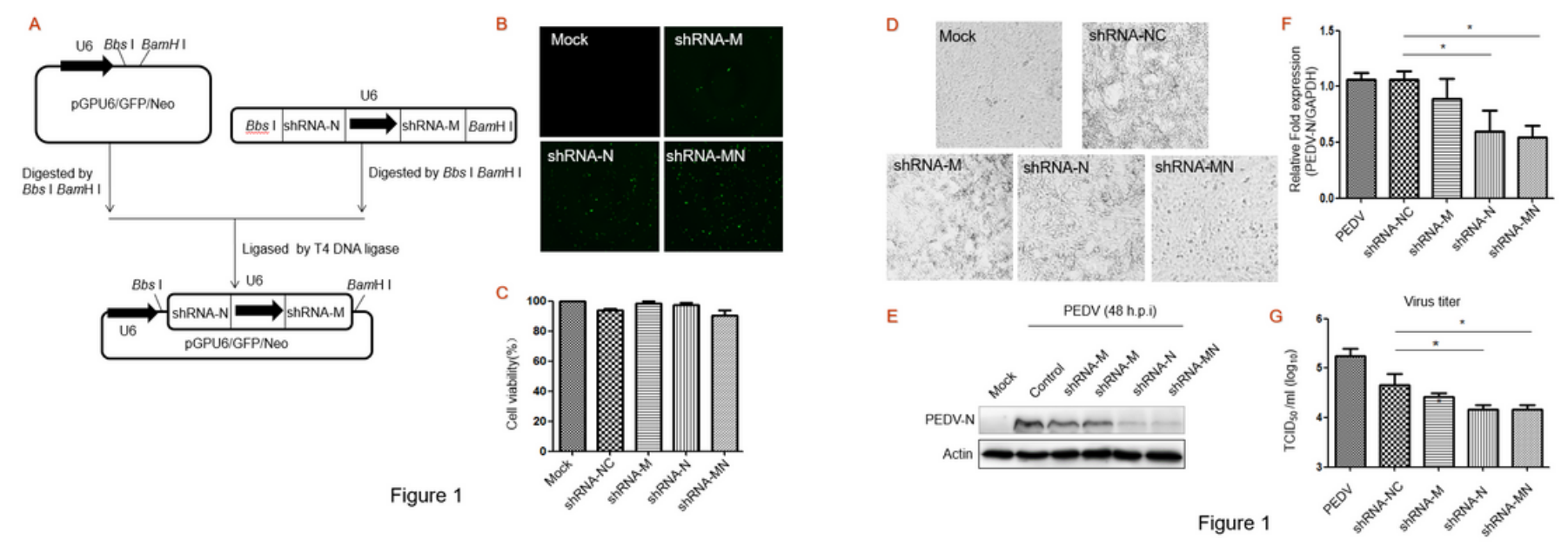

\section{Figure 1}

The design of shRNA-expressing plasmids and antiviral activity A, Schematic illustration of construction of the dual shRNA expression plasmid (pGPU6/GFP/Neo-shRNA-MN). B, Cells were transfected with shRNA-expressing plasmids for $24 \mathrm{~h}$, transfection efficiency was observed using fluorescence microscope. C, The effect on cell viability was assessed by MTT assay. D, Cytopathic effects caused by PEDV infection. Vero cells were transfected with shRNA-expressing plasmids for $24 \mathrm{~h}$, and then infected with PEDV at $0.1 \mathrm{MOI}$. At $24 \mathrm{~h}$ post-infection, cytopathic effects were observed using microscope. E-G, At $48 \mathrm{~h}$ post-infection, cells were harvested and immunoblotted with antibody to viral protein PEDV-N, with actin as a control. N/A, the change in abundance of PEDV-N was analyzed by densitometry analysis using Image-Pro Plus Software and normalized to actin. Under the same experimental conditions, total RNA was isolated and PEDV mRNA was quantified by qRT-PCR normalized against GAPDH (F), and viral yields in the medium were determined by TCID50 (G). These experiments were performed two times with three replicates in each experiment. Significance was determined by one-way analysis. Values represent means and SD for triplicates, ${ }^{\star} p<0.05$. 


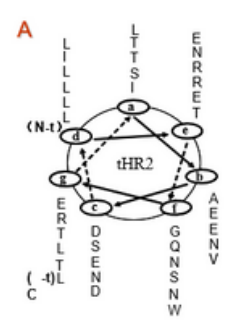

C

HR1:

NSAIGNITSAFESVKEAISQTSKG LNTVAHALTKVQEV VNSQGSA LNQLTVQ LOHNFQA

efg abcdefg abcdefg abcdefg abcdefg abcdefg abcdefg abcdefg abcdefg

tHR2:

LTGE IADLEQR SESLRNT TEELRSLINNINNT LVDLEWL.

defg abcdefg abcdefg abcdefg abcdefg abcdefg

B

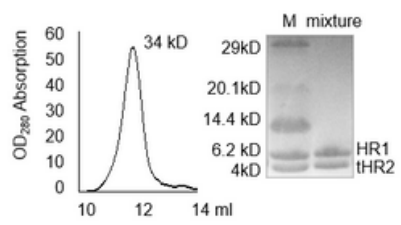

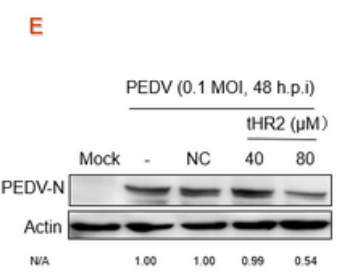

D

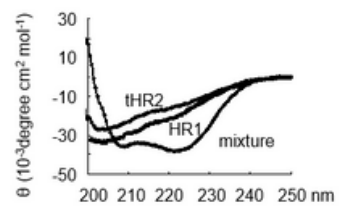

Figure 2
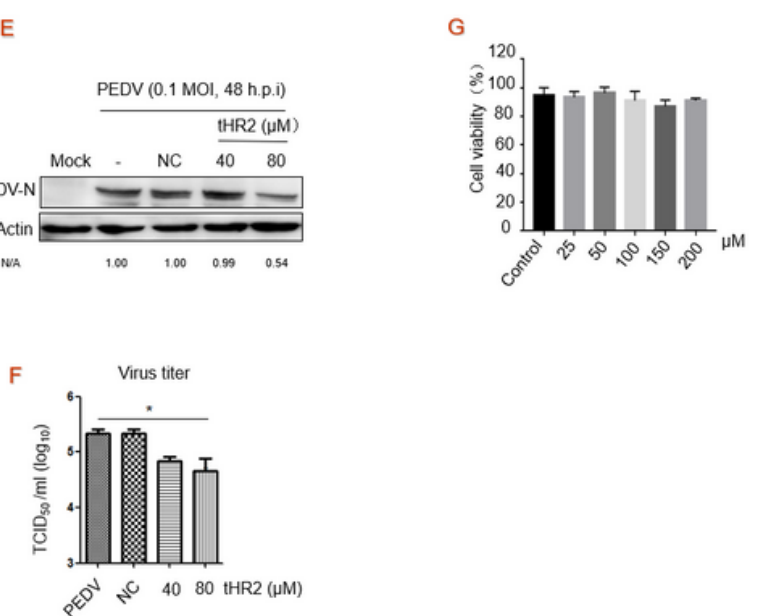

Figure 2

\section{Figure 2}

Construction and antiviral activity of soluble peptide tHR2 A, The primary structure of each helix consists of heptad repeats (HRs) $(a-b-c-d-e-f-g) n$, in which $a, d$, and e are interactive sties and b, c, f, and $g$ are solvent-accessible sites. Accordingly, the region of residues from 1263 to 1314 (52 residues) is the potential HR2 domain. The truncated HR2 (tHR2) domain contains residues 1278 to 1316 (39 residues). $\mathrm{B}$, Gel-filtration analysis of combination of HR1 and tHR2 at the equimolar concentration. C, The primary structure of each a-helix consists of a heptad repeat composed of seven residues commonly denoted by $(a-b-c-d-e-f-g) n$, in which $a, d$, and e are interactive sties. Most important helix-forming residues of site a are highly conserved leucine $(L)$ or isoleucine $(I)$ residues; residues of sites $d$ and e are relatively conserved. D, Circular dichroism (CD) spectra analysis. A typical a-helix secondary structure for mixing HR1 and tHR2 was showed. HR1, tHR2 and mixture of both were diluted in PBS for indicated concentrations. The wavelength-dependence of molar ellipticity is monitored at $25^{\circ} \mathrm{C}$ as the average of eight scans in a spectropolarimeter. E and F, Vero cells were treated with scramble (NC) or tHR2 peptides at concentrations of 40 and $80 \square \mathrm{M}$ at the time of PEDV infection for $1 \mathrm{~h}$ at $37^{\circ} \mathrm{C}$, washed three times with PBS, and overlaid with DMEM-2\% FBS containing tHR2. At $48 \mathrm{~h}$ post infection, cells were harvested, and viral proteins were detected by western blotting and viral titers were evaluated by TCID50 assay. G, Effects of tHR2 on cell viability. Cells were treated with various concentrations of tHR2 for $24 \mathrm{~h}$, and cell viability was determined by MTT assay. These experiments were performed two times with three replicates in each experiment. Significance was determined by one-way analysis. Values represent means and SD. ${ }^{*} p<0.05$ compared to PBS-treated group. 
A
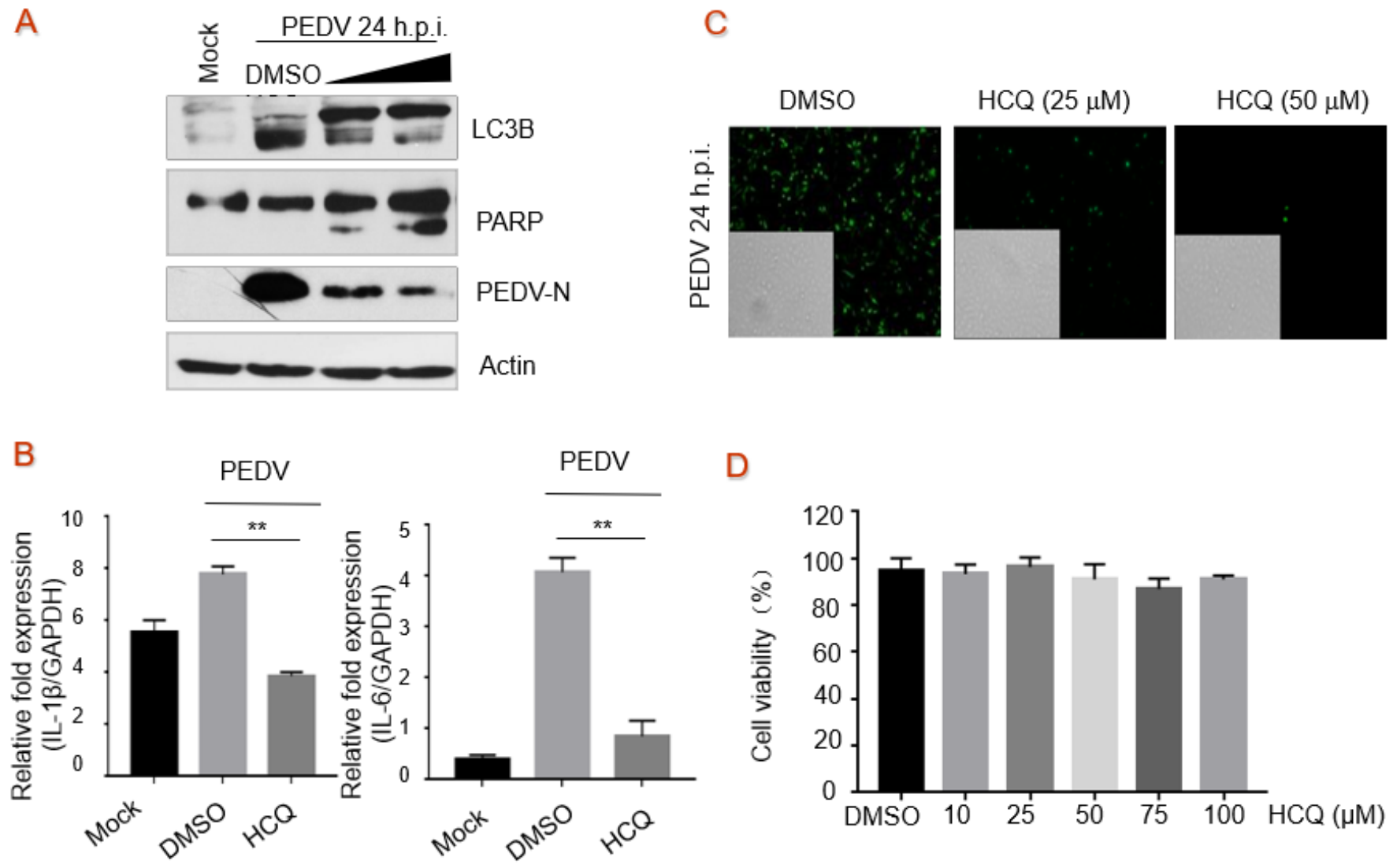

Figure 3

\section{Figure 3}

The effect of HCQ on autophagy and inflammatory response A, Vero cells were treated with HCQ at the time of infection for $1 \mathrm{~h}$ at $37^{\circ} \mathrm{C}$, washed three times with PBS, and overlaid with DMEM-2\% FBS containing HCQ, and LC3B, PARP, and PEDV-N were measured by immunoblotting with specific antibodies, with actin as a control. B, Vero cells were mock-infected or infected with PEDV at $0.1 \mathrm{MOI}$ in the presence of the DMSO or HCQ at $50 \mathrm{aM}$ for $24 \mathrm{~h}$, total RNAs were isolated and expression levels of IL-

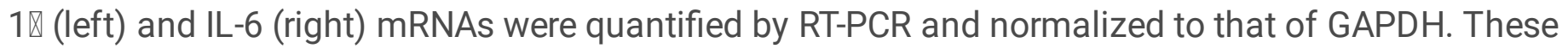
experiments were performed two times with three replicates in each experiment. Significance was determined by one-way analysis of variance (ANOVA) with Dennett's multiple-comparison test. Values represent means and SD, * $p<0.05$. C, Vero cells were infected with PEDV of $0.1 \mathrm{MOI}$ in the presence of $\mathrm{HCQ}$ at 25 and $50 \square \mathrm{M}$ for $24 \mathrm{~h}$. The infected cells were imaged by fluorescence microscopy using an Olympus IX73 microscope (magnification of $100 \times$ ) equipped with a DP73 camera, and inserted picture is assessed under light microscopy. D, Cell viability was assessed by MTT assay according to the manufacturer's protocols. Each image is representative of three experiments. 

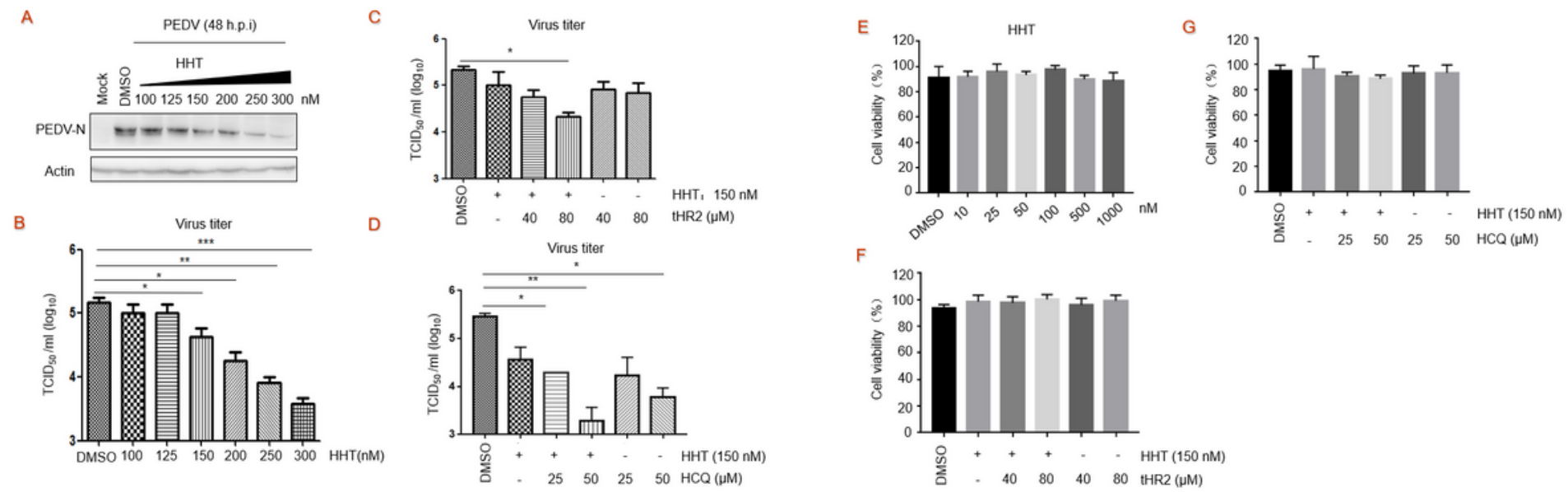

Figure 4

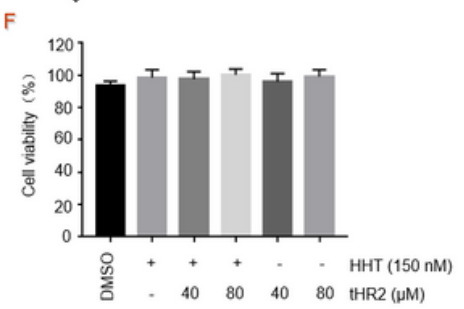

Figure 4

\section{Figure 4}

Antiviral activity of combination treatment A-B, Vero cells seeded at $2.5 \times 106$ cells per well in six-well plates were infected with PEDV at $0.1 \mathrm{MOI}$ in the presence of the HHT at different concentrations for $48 \mathrm{~h}$, electrophoretically separated proteins were analyzed by immunoblotting with antibodies to viral protein PEDV-N and actin (A). Viral yields in the medium were determined by TCID50 (B). These experiments were performed two times with three replicates in each experiment. Values represent means and SD. ${ }^{*}<0.05$; $\star \star p<0.01 ; * \star \star p<0.001$ compared to PBS-treated group. C-D, Vero cells seeded at $2.5 \times 106$ cells per well in six-well, cells were then infected with PEDV at $0.1 \mathrm{MOI}$ in the presence of peptide tHR2 and HHT (C) or HCQ (D). At $48 \mathrm{~h}$ post-infection, virus titer in TCID50 was determined. E-G, Cytotoxicity of HHT and combination treatment was detected by MTT assay. These experiments were performed two times with three replicates in each experiment. Values represent means and SD for triplicates. ${ }^{*} p<0.05,{ }^{\star \star} p<0.01$ for one-way ANOVA.. 